\title{
Presença ou ausência dos pais durante o atendimento odontológico: revisão integrativa
}

\author{
Parental presence/absence in dental operatory: integrative review
}

Erika Yukari Azechi Paz ${ }^{1}$, Cintia Regina Tornisiello Katz ${ }^{1 *}$

\begin{abstract}
RESUMO
Este estudo objetivou avaliar as evidências científicas disponíveis acerca da influência da presença ou ausência dos pais no comportamento infantil, durante o atendimento odontológico. Realizou-se uma revisão integrativa da literatura através da busca sistematizada nas bases de dados: PUBMED, MEDLINE, LILACS, BBO e SciELO. Como critérios de busca foram utilizadas as palavras "presence", "absence", "parental", "dental" e "treatment", sem restrições de campo de aparecimento dessas palavras nos artigos. Foram selecionados inicialmente 42 artigos, dos quais, após a leitura dos títulos e a exclusão das repetições, totalizaram 10 artigos. Foram excluídos dois artigos por não estarem mais disponíveis no meio eletrônico. Assim, oito artigos fizeram parte desta revisão. Destes, cinco estudos objetivaram avaliar a influência da presença ou ausência dos pais no comportamento infantil durante as consultas e três avaliaram a efetividade da técnica PPA (Parental Presence/Ausence) em crianças não colaboradoras. Seis estudos foram do tipo ensaio clínico. As evidências científicas disponíveis mostram que não existem diferenças no comportamento infantil na presença ou ausência materna durante o tratamento odontológico.
\end{abstract}

Palavras-chave: Comportamento Infantil; Relação Dentista-Paciente; Relação Pais-Filhos; Tratamento Odontológico.

\begin{abstract}
This study aimed to assess the available scientific evidence on the influence of parental presence/absence on children behavior during dental treatment. An integrative literature review was conducted by a systematized search in the PUBMED, MEDLINE, LILACS, BBO and SciELO databases. The words "presence", "absence", "parental", "dental" and "treatment" were used as advanced standard search, without restriction of field appearing in the articles. Forty-two articles were initially selected, ten articles were remained after reading the titles and exclusion of the repeated. Those, two articles were excluded because they were no available in the electronic literature. Thus, eight articles composed this study. It was observed that five articles assessed the influence of parental presence/absence on children behavior during dental treatment, and three articles assessed the effectiveness of PPA (Parental Presence/Ausence) technique on uncooperative children. Six of eight selected articles were clinical trials. According to the present study there are no difference on parental presence/absence in children behavior management during the dental treatment.
\end{abstract}

Keywords: Children Behavior; Dentist-Patient Relation; Parent-Child Relations; Dental Treatment.

\footnotetext{
${ }^{1}$ Universidade Federal de Pernambuco

*E-mail: cintia.katz@ufpe.br
} 


\section{INTRODUÇÃO}

As crianças encontram-se em um processo contínuo de crescimento físico e mental, no qual cada faixa etária tem várias transformações no comportamento. Os profissionais que atendem crianças devem estar aptos para abordá-las, por meio do conhecimento das técnicas de gerenciamento comportamental indicadas para cada fase, com o objetivo de criar confiança e dissipar medos e ansiedades que podem estar presentes durante as consultas (SATO e CORRÊA, 2017; AAPD, 2019).

Existem técnicas básicas de gerenciamento comportamental, fundamentadas na comunicação com o paciente, como "dizer-mostrar-fazer", "modelagem”, "distração" e "reforço positivo"; como também existem técnicas avançadas (SATO e CORRÊA, 2017; AAPD, 2019). Estas últimas são utilizadas quando não houver sucesso na colaboração do paciente com a utilização das técnicas básicas (SATO e CORRÊA, 2017). Em Odontopediatria, espera-se encontrar $10 \%$ dos pacientes com problemas no manejo do comportamento infantil (KLINGBERG e BROBERG, 2007).

Os pais desempenham um papel importante no comportamento dos filhos durante o atendimento odontológico e por essa razão sua influência permanece como um tópico de grande relevância em Odontopediatria. Enquanto a presença dos pais durante as consultas médicas é bastante comum, muito tem se discutido na literatura sobre os efeitos da presença ou ausência parental no comportamento infantil durante o tratamento odontológico (FENLON, DABBS e CURZON, 1993; KOTSANOS, ARHAKIS e COOLIDGE, 2005; KOTSANOS, et al. 2009; COX, KRIKKEN e VEERKAMP, 2011; BOKA, et al. 2017; AAPD, 2008).

Alguns autores defendem a presença dos pais nas consultas de crianças muito jovens, relatando vantagens na presença parental para transmitir segurança e servir de modelo de comportamento para os filhos em situações desconhecidas (FENLON, DABBS e CURZON, 1993; COX, KRIKKEN e VEERKAMP, 2011). Outros autores relatam diversos motivos para a exclusão dos pais no consultório odontológico, como o aumento dos problemas de manejo de comportamento, o reforço de comportamentos aversivos durante os procedimentos e a possibilidade de projeção de sentimentos como ansiedade e medo dos pais nas crianças (FENLON, DABBS e CURZON, 1993; KOTSANOS, et al. 2009; BOKA, et al. 2017). A Academia Americana de Odontopediatria reconhece que nos casos de comportamento extremamente negativo a 
presença dos pais pode reforçar o mau comportamento e dificultar o estabelecimento de comunicação efetiva entre dentista e paciente (AAPD, 2019).

Tem sido observado, na prática clínica atual e em estudos recentes, que a maioria dos pais não quer se separar de seus filhos durante os procedimentos odontológicos (AAPD, 2019; COX, KRIKKEN e VEERKAMP, 2011; VASILIKI, et al. 2016; AHUJA, et al. 2018). De acordo com esses estudos, as possíveis razões para isto estão relacionadas com a idade cada vez mais precoce em que as crianças iniciam suas consultas odontológicas, com o próprio desejo dos pais da atualidade, ou com o fato de a Odontopediatria estar utilizando abordagens cada vez mais preventivas, necessitando do maior envolvimento dos pais.

A negociação da presença ou ausência parental durante as consultas odontológicas tem sido considerada entre as técnicas avançadas de manejo do comportamento (SATO e CORRÊA, 2017; AAPD, 2019). Chamada de PPA (Parental Presence/Absence), esta técnica consiste em pedir para que os pais saiam do ambiente odontológico, no caso de comportamento negativo da criança, como uma forma de restabelecer a comunicação. Com a saída dos pais, o dentista tem a atenção da criança voltada exclusivamente para as suas orientações, visando a melhora do seu comportamento. Feito isso, e quando a criança passa a colaborar com os procedimentos, os pais são solicitados a retornar ao ambiente (AAPD, 2019; KOTSANOS, ARHAKIS e COOLIDGE, 2005; KOTSANOS, et al. 2009; BOKA, et al. 2017).

Pelo exposto, verifica-se que a presença ou ausência parental durante o tratamento odontológico tem sido um assunto controverso (COX, KRIKKEN e VEERKAMP, 2011). Assim, visando analisar as evidências científicas a respeito desta temática, este trabalho objetivou realizar uma revisão integrativa da literatura sobre a influência da presença ou ausência dos pais no comportamento infantil durante o atendimento odontológico.

\section{MATERIAIS E MÉDODOS}

Trata-se de uma busca sistematizada da literatura científica nas bases de dados: PUBMED (United States National Library of Medicine), MEDLINE (Literatura Internacional em Ciências da Saúde), LILACS (Literatura Latino-Americana e do Caribe em Ciências da Saúde), BBO (Bibliografia Brasileira de Odontologia) e SciELO (Sciece Eletronic Library Online). Em todas as bases as buscas foram realizadas utilizando-se os formulários de busca avançada. 
Como estratégia de busca foi utilizada a seguinte sequência e combinação de palavras, com restrição de aparecimento no título/resumo dos artigos científicos indexados: "presence" OR “absence" AND "parental” AND “dental” AND "treatment". Estes critérios possibilitaram a seleção inicial de 84 artigos (Figura 1), sendo: 41 artigos na PUBMED; 37 no MEDLINE, 01 na BBO, 01 na LILACS e 01 na base SciELO. Não houve restrição no ano de publicação, idioma ou faixa etária dos estudos.

A partir desta seleção inicial, foram excluídos os trabalhos em duplicidade nas bases de dados, procedendo-se à leitura de todos os títulos e/ou resumos (rastreamento). Para esta etapa foram selecionados os trabalhos que atenderam ao seguinte critério de elegibilidade: estudos com amostras clínicas que avaliaram o comportamento infantil de acordo com a presença ou ausência dos pais durante as consultas odontológicas. Dez artigos atenderam ao critério de elegibilidade, sendo dois excluídos por não estarem mais disponíveis em meio eletrônico. Sendo assim, oito estudos foram considerados para a análise qualitativa deste trabalho

\section{RESULTADOS E DISCUSSÃO}

A partir dos critérios metodológicos adotados, foram considerados para esta revisão, oito artigos, os quais se encontram sumarizados no Quadro 1, por ordem cronológica. Destaca-se que os estudos foram realizados nos seguintes países: Grécia, Holanda, Arábia Saudita e Índia. 
Quadro 1: Estudos selecionados de acordo com autor, ano, amostra, faixa etária, objetivo, métodos, instrumentos e principais resultados.

\begin{tabular}{|c|c|c|c|c|}
\hline $\begin{array}{c}\text { Autor/ } \\
\text { Ano/Local }\end{array}$ & $\begin{array}{c}\text { Amostra e Faixa } \\
\text { Etária }\end{array}$ & Objetivo & Métodos*/ Instrumentos & Principais Resultados \\
\hline $\begin{array}{l}\text { Kotsanos, } \\
\text { Arkhakis, } \\
\text { Coolidge } \\
\text { (2005) } \\
\text { GRÉCIA }\end{array}$ & $\begin{array}{l}170 \text { crianças } \\
\text { de } 3 \text { a } 10 \text { anos: } \\
-85 \text { não } \\
\text { colaboradoras } \\
-85 \text { controles. }\end{array}$ & $\begin{array}{l}\text { Avaliar a efetividade da } \\
\text { técnica PPA em } \\
\text { pacientes não } \\
\text { colaboradores. }\end{array}$ & $\begin{array}{l}\text { Estudo retrospectivo } \\
\text { Procedimentos } \\
\text { restauradores e } \\
\text { cirúrgicos } \\
\text { Escala de Frankl }\end{array}$ & $\begin{array}{l}\text { A técnica PPA obteve } \\
\text { sucesso de } 94 \% \text { com } \\
\text { estatística significante em } \\
\text { crianças inicialmente não } \\
\text { colaboradoras. }\end{array}$ \\
\hline $\begin{array}{l}\text { Kotsanos et } \\
\text { al. (2009) } \\
\text { GRÉCIA }\end{array}$ & $\begin{array}{l}440 \text { crianças } \\
\text { de } 3 \text { a } 10 \text { anos: } \\
-75 \text { não } \\
\text { colaboradoras e } \\
365 \text { colaboradoras }\end{array}$ & $\begin{array}{l}\text { Avaliar a efetividade } \\
\text { da técnica PPA em } \\
\text { pacientes não } \\
\text { colaboradores. }\end{array}$ & $\begin{array}{l}\text { EC não randomizado } \\
\text { Procedimentos } \\
\text { restauradores e } \\
\text { cirúrgicos. } \\
\text { Escala de Frankl }\end{array}$ & $\begin{array}{l}\text { A técnica PPA obteve } \\
\text { sucesso de } 93.3 \% \text { com } \\
\text { estatística significante em } \\
\text { crianças inicialmente não } \\
\text { colaboradoras. }\end{array}$ \\
\hline $\begin{array}{l}\text { Cox et al. } \\
(\mathbf{2 0 1 1 )} \\
\text { HOLANDA }\end{array}$ & $\begin{array}{l}90 \text { crianças } \\
\text { de } 4 \text { a } 12 \text { anos: } \\
-47 \text { com os pais } \\
-43 \text { sem os pais }\end{array}$ & $\begin{array}{l}\text { Analisar a influência da } \\
\text { presença dos pais no } \\
\text { comportamento infantil } \\
\text { durante o tratamento } \\
\text { odontológico. }\end{array}$ & $\begin{array}{l}\text { ECR } \\
\text { Pelo menos duas sessões. } \\
\text { Escala Venham } \\
\text { (dentista) e Wong-Baker } \\
\text { (criança) }\end{array}$ & $\begin{array}{l}\text { Para as crianças, não } \\
\text { influenciou no tratamento. } \\
\text { Dentistas relataram } \\
\text { desvantagens na presença } \\
\text { parental em crianças } \\
\text { ansiosas. }\end{array}$ \\
\hline $\begin{array}{l}\text { Vasiliki et } \\
\text { al. (2016) } \\
\text { GRÉCIA }\end{array}$ & $\begin{array}{l}\mathbf{1 0 0} \text { crianças } \\
\text { de } 4 \text { a } 12 \text { anos: } \\
-50 \text { com os pais } \\
-50 \text { sem os pais }\end{array}$ & $\begin{array}{l}\text { Analisar a influência da } \\
\text { presença dos pais no } \\
\text { comportamento infantil } \\
\text { durante o atendimento } \\
\text { odontológico. }\end{array}$ & $\begin{array}{l}\text { ECR } \\
\text { Escala de Venham } \\
\text { (dentista e pais) } \\
\text { Escala de Wong-Baker } \\
\text { (criança) }\end{array}$ & $\begin{array}{l}\text { A presença/ausência } \\
\text { parental não influenciou no } \\
\text { comportamento. Crianças } \\
\text { relataram desconfortos. } \\
\text { Dentistas e responsáveis } \\
\text { reportaram prejuízos no } \\
\text { comportamento infantil. }\end{array}$ \\
\hline $\begin{array}{l}\text { Sharat et } \\
\text { al. (2016) } \\
\text { ARÁBIA } \\
\text { SAUDITA }\end{array}$ & $\begin{array}{l}\mathbf{1 5 0} \text { crianças } \\
\text { de } 6 \text { e } 8 \text { anos: } \\
-50 \text { com pais } \\
-50 \text { com mães } \\
-50 \text { sem mães/pais }\end{array}$ & $\begin{array}{l}\text { Avaliar a influência da } \\
\text { presença dos pais no } \\
\text { durante o atendimento } \\
\text { odontológico. }\end{array}$ & $\begin{array}{l}\text { EC não randomizado } \\
\text { Aferição do ritmo } \\
\text { cardíaco: oxímetro; } \\
\text { Procedimentos } \\
\text { restauradores } \\
\text { Escala de Venham }\end{array}$ & $\begin{array}{l}\text { A presença dos pais } \\
\text { reduziu as manifestações } \\
\text { fisiológicas da ansiedade, } \\
\text { mas não houve diferença } \\
\text { no comportamento infantil. }\end{array}$ \\
\hline $\begin{array}{l}\text { Boka } \text { et al. } \\
2017 \\
\text { GRÉCIA }\end{array}$ & $\begin{array}{l}\mathbf{6 1} \text { crianças } \\
\text { de } 3 \text { a } 8 \text { anos, } \\
\text { não colaboradoras }\end{array}$ & $\begin{array}{l}\text { Avaliar a efetividade da } \\
\text { técnica PPA em crianças } \\
\text { não colaboradoras. }\end{array}$ & $\begin{array}{l}\text { ECR } \\
\text { Procedimentos } \\
\text { restauradores e } \\
\text { cirúrgicos. } \\
\text { Escala de Frankl }\end{array}$ & $\begin{array}{l}\text { A técnica PPA não se } \\
\text { mostrou superior às outras } \\
\text { técnicas não } \\
\text { farmacológicas de manejo } \\
\text { do comportamento. }\end{array}$ \\
\hline $\begin{array}{l}\text { Ahuja et al. } \\
(\mathbf{2 0 1 8 )} \\
\text { ÍNDIA }\end{array}$ & $\begin{array}{l}\mathbf{3 0} \text { crianças } \\
\text { de } 4 \text { a } 7 \text { anos }\end{array}$ & $\begin{array}{l}\text { Avaliar a influência da } \\
\text { presença dos pais } \\
\text { durante o atendimento } \\
\text { odontológico. }\end{array}$ & $\begin{array}{l}\text { EC não randomizado } \\
1^{\text {a }} \text {. sessão na presença, } \\
2^{\text {a }} \text {. sessão na ausência. } \\
\text { Procedimentos } \\
\text { restauradores. } \\
\text { Escala de Frankl }\end{array}$ & $\begin{array}{l}\text { Não houve diferença } \\
\text { significativa no } \\
\text { comportamento da criança } \\
\text { na presença/ausência dos } \\
\text { pais. }\end{array}$ \\
\hline $\begin{array}{l}\text { Archarya, } \\
\text { Priyanka, } \\
\text { Archarya, } \\
\text { (2019) } \\
\text { ÍNDIA }\end{array}$ & $\begin{array}{l}\mathbf{6 0} \text { crianças } \\
\text { de zero a } 12 \text { anos }\end{array}$ & $\begin{array}{l}\text { Avaliar a influência da } \\
\text { presença dos pais } \\
\text { durante o atendimento } \\
\text { odontológico. }\end{array}$ & 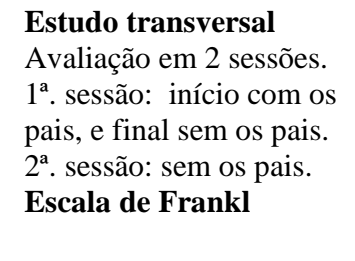 & $\begin{array}{l}\text { Nas crianças maiores de } 6 \\
\text { anos, não houve diferença } \\
\text { significante no } \\
\text { comportamento. Crianças } \\
\text { mais novas mostraram } \\
\text { comportamento negativo } \\
\text { na ausência dos pais. }\end{array}$ \\
\hline
\end{tabular}

*EC: Ensaio Clínico. ECR: Ensaio Clínico Randomizado 
A partir da análise dos objetivos dos estudos selecionados, observa-se que cinco deles avaliaram a influência da presença ou ausência dos pais no comportamento infantil durante as consultas odontológicas (COX, KRIKKEN e VEERKAMP, 2011; VASILIKI, et al. 2016; AHUJA, et al. 2018; ARCHARYA, PRIYANKA e ARCHARYA, 2019; SHARAT, et al. 2016) e três estudos avaliaram a efetividade da técnica PPA em crianças não colaboradoras (KOTSANOS, ARHAKIS e COOLIDGE, 2005; KOTSANOS, et al. 2009; BOKA, et al. 2017; AAPD, 2008).

Considerando os desenhos de estudo dos trabalhos que avaliaram a presença ou ausência parental no comportamento infantil durante as consultas odontológicas, três foram do tipo ensaio clínico randomizado (COX, KRIKKEN e VEERKAMP, 2011; VASILIKI, et al. 2016; SHARAT, et al. 2016), um estudo foi do tipo ensaio clínico não randomizado (AHUJA, et al. 2018) e um estudo foi classificado como transversal (ARCHARYA, PRIYANKA e ARCHARYA, 2019). Observou-se que os referidos ensaios clínicos utilizaram como instrumentos, as escalas de Venham (VENHAM, et al. 1988) e de Wong Baker (WONG e BAKER, 1988). Os demais utilizaram a escala de Frankl (FRANKL, SHIERE e FOGELS, 1962).

Para analisar e comparar esses estudos é necessário observar o que cada escala avalia. A escala de Venham classifica o comportamento das crianças durante o tratamento odontológico em cinco escores, considerando detalhadamente as reações da criança como choro e/ou protestos físicos (VENHAM, et al. 1980). A escala de Wong Baker é uma escala de faces, que consiste em seis faces desenhadas expressando do sorriso ao choro, na qual os pacientes podem classificar o seu nível de percepção, é frequentemente utilizada para expressar dor e desconforto (WONG e BAKER, 1988). A escala de Frankl consiste em quatro classificações do comportamento infantil nas consultas odontológicas, que variam do comportamento definitivamente negativo ao definitivamente positivo (FRANKL, SHIERE e FOGELS, 1962). Essa última escala apresenta critérios muito semelhantes à escala de Venham, porém com menos escores, sendo de mais fácil utilização.

Nos ensaios clínicos supramencionados, quando houve randomização (COX, KRIKKEN e VEERKAMP, 2011; VASILIKI, et al. 2016), esta foi em relação à determinação (aleatória) das crianças que ficariam ou não com os pais durante as consultas. Todos os ensaios clínicos foram realizados com amostras entre 90 a 150 crianças, com exceção do estudo de Ahuja et al. (2018) (n=30). Os dois ensaios clínicos 
randomizados (COX, KRIKKEN e VEERKAMP, 2011; VASILIKI, et al. 2016) foram realizados com amostras semelhantes em número e faixa etária, e com os mesmos instrumentos, avaliando as percepções das crianças e dos dentistas; ambos concordaram nos achados de que não houve diferença significativa no comportamento infantil na presença ou ausência dos pais.

O ensaio clínico realizado por Sharat et al. (2016) $(\mathrm{n}=150)$ avaliou ritmo cardíaco das crianças durante os procedimentos de anestesia e restauradores, o que foi atribuído como um indicador fisiológico de ansiedade, bem como o comportamento das mesmas durante as consultas. Neste estudo não houve randomização. Os autores registraram que no grupo de crianças acompanhadas pelos pais (figura paterna), a maioria era do sexo masculino, enquanto, no grupo acompanhado pelas mães, a maioria das crianças era do sexo feminino. Este fato foi justificado por ser uma questão cultural da população saudita. A perda amostral foi maior no grupo de crianças acompanhadas pelos pais, o que trouxe limitações tanto na análise da presença da figura paterna, como na análise das respostas das crianças do sexo masculino. Este estudo revelou que as crianças atendidas na presença parental apresentaram menores alterações no ritmo cardíaco que as atendidas na ausência parental, entretanto não houve alterações no comportamento durante as consultas na presença ou ausência parental.

Pelo exposto, mesmo considerando essas diferenças e/ou limitações, todos ensaios clínicos considerados nesta primeira análise (COX, KRIKKEN e VEERKAMP, 2011; VASILIKI, et al. 2016; AHUJA, et al. 2018; SHARAT, et al. 2016) concordaram no resultado de que a presença ou ausência dos pais não influenciou no comportamento infantil durante os atendimentos.

Com relação ao estudo de Archarya, Pryanka e Archariya (2019) (n=60), os próprios autores o classificaram como de natureza transversal, porém o comportamento infantil foi comparado em duas sessões, sendo que, na primeira, alguns pais iniciavam as consultas acompanhando as crianças e após "algum tempo" os pais eram solicitados a sair da sala; e na segunda, os pais não estavam presentes. Nesse estudo, a amostra de 60 pacientes é considerada pequena, dada a amplitude da faixa etária amostral, e o desenho metodológico é considerado frágil para os objetivos propostos.

Considerando os três estudos que avaliaram a efetividade da técnica PPA Kotsanos, Arkhakis e Coolidge (2005), Kotsanos et al. (2009) e Boka et al. (2017), 
ressalta-se que os mesmos foram realizados em sequência, na Grécia (cidade de Tessalônica). Destes, Kotsanos et al. (2009) e Boka et al. (2017) foram ensaios clínicos, havendo randomização apenas neste último. ${ }^{9}$

O estudo de Kotsanos, Arkhakis e Coolidge (2005) foi um estudo preliminar, retrospectivo, realizado com base nos dados de prontuários das crianças atendidas em uma clínica privada ( $\mathrm{n}=170$, faixa etária= 3 a 10 anos), cuja avaliação do comportamento dos pacientes infantis pela Escala de Frankl era feita de rotineiramente, por todos os odontopediatras, nas crianças acima de 3 anos. Já o estudo de Kotsanos et al. (2009) foi um ensaio clínico realizado com 440 crianças, da mesma faixa etária do estudo anterior (3 a 10 anos), sendo na mesma clínica privada do anterior. Esses dois estudos observaram um alto percentual de sucesso da técnica PPA em crianças não colaboradoras.

Um aspecto importante a ser ressaltado na metodologia desses dois estudos (KOTSANOS, ARKHAKIS e COOLIDGE, 2005; KOTSANOS, et al. 2009) é que todos os pais eram convidados a permanecer na sala de atendimento no início das consultas. Entretanto, se o comportamento negativo fosse observado, o dentista interrompia o atendimento e explicava para a criança a política da presença parental adotada pela clínica. De acordo com os autores dos dois estudos, isto era feito de maneira muito empática. Em seguida os pais eram solicitados a dar o seu consentimento oral, caso eles fossem solicitados a sair da sala. Se o comportamento negativo continuasse, os pais eram solicitados a sair para aguardar na sala de espera, e o dentista lembrava a criança de que os pais retornariam assim que ela parasse de chorar. Quando a criança parava de chorar, o dentista verbalmente reforçava a criança e imediatamente solicitava a presença dos pais de volta na sala de atendimento.

No estudo de Boka et al. (2017) houve maior rigor metodológico, comparativamente aos supracitados (KOTSANOS, ARKHAKIS e COOLIDGE, 2005; KOTSANOS, et al. 2009), tendo sido realizado apenas com crianças não colaboradoras, ou seja, que apresentaram escores 1 ou 2 de Frankl. A amostra contou com 61 crianças de 3 a 8 anos de idade, sendo 31 do grupo caso (aplicação da técnica PPA) e 30 do grupo controle. Os tratamentos foram conduzidos por 6 pós-graduandas consideradas experientes (concluintes) e cada uma foi instruída a usar as mesmas palavras, de maneira empática, quando da explicação da técnica às crianças. As sessões foram gravadas e assistidas por um avaliador cego em relação à técnica utilizada. No grupo controle foram utilizadas outras técnicas de manejo comportamental não farmacológico. Técnicas 
aversivas ou restritivas não foram utilizadas em nenhum paciente da pesquisa. Nesse estudo, a técnica PPA não se mostrou superior às demais técnicas não farmacológicas.

A técnica PPA passou a ser reconhecida pela Academia Americana de Odontopediatria a partir de 2008, como uma das técnicas de manejo de comportamento infantil não farmacológico, sendo a segunda técnica mais aceita, depois da técnica “dizermostrar-fazer". Atualmente, a utilização da PPA é considerada como uma maneira consentida de agir, de modo rápido e fácil, às mudanças no curso do tratamento ou na orientação do comportamento infantil. É uma técnica destinada principalmente a crianças que tenham comportamento desafiador e que não aceitem colaborar com o tratamento, indicada por muitos autores como uma técnica de recompensa nos casos em que o paciente permita uma comunicação mais efetiva com o profissional (KOTSANOS, ARKHAKIS e COOLIDGE, 2005; KOTSANOS, et al. 2009; BOKA et al. 2017).

Considerando a análise dos artigos que compuseram esta revisão, pode-se afirmar que as evidências científicas disponíveis mostram que não existem diferenças no comportamento infantil na presença ou ausência parental.

Deve-se ressaltar que as diversidades culturais e sociais entre os países pode interferir na escolha das técnicas de administração do comportamento infantil adotadas pelos cirurgiões-dentistas em todo mundo, assim como no comportamento dos pais e crianças. Considerando os países em que os estudos desta revisão foram realizados (Grécia, Holanda, Arábia Saudita e Índia), pode-se sugerir futuros com outras populações, como por exemplo a latina e americana, a fim de observar os efeitos das diversidades culturais e sociais nos achados.

Na realização de outros ensaios clínicos sobre a efetividade da técnica PPA, sugere-se considerar como critério de seleção as idades acima de seis anos, uma vez que as crianças abaixo desta idade podem sofrer ansiedade de separação dos pais (KOTSANOS, ARKHAKIS e COOLIDGE, 2005; COX, KRIKKEN e VEERKAMP, 2011), sendo este um fator a ser considerado na avaliação do comportamento infantil.

O presente estudo apresenta como limitações: a adoção de critérios de busca menos rigorosos que os utilizados em uma revisão sistemática, a inclusão de estudos disponíveis apenas em formato eletrônico, e a não utilização de instrumento de análise da qualidade dos artigos. 


\section{CONCLUSÕES}

A análise das evidências científicas disponíveis, considerando os limites de uma revisão integrativa, permitiu concluir que não existe diferença no comportamento infantil durante o tratamento odontológico na presença ou ausência dos pais.

\section{REFERÊNCIAS}

AAPD. American Academy of Paediatric Dentistry. Clinical Affairs Committee, Guideline on behavior Guidance for the paediatric dental patient. The Reference Manual of Pediatric Detistry, v. 29, n. 1, p.115-124, 2008.

AAPD (American Academy of Pediatric Dentistry). Guideline on behavior guidance for the pediatric dental patient. Journal of American Dental Association, v.40, n. 1, p. 254-267, 2019.

AHUJA, S.; GANDHI, K.; MALHOTRA, R.; KAPOOR, R.; MAYWAD, S.; DATTA, G. Assessment of the effect of parental presence in dental operatory on the behavior of children aged 4-7 years. Journal of Indian Society of Pedodontics and Preventive Dentistry, v. 36, n. 2, p. 167-72, 2016.

ARCHARYA, S.; PRIYANKA, J.; ARCHARYA, S. Parental presence in dental operatory as a behavior management tool on children in Bhubaneswar. Pesquisa Brasileira em Odontopediatria e Clínica Integrada, v.19, n. 1, p. e4506, 2019.

BOKA V, ARAPOSTATHIS K, CHARITOUDIS G, VEERKAMP J, VAN LOVEREN C, KOTSANOS N. A study of parental presence/ absence technique for child dental behaviour management. European Archives of Paediatric Dentistry, v.18, n. 6, p. 405-09, 2017.

COX, I. C.; KRIKKEN, J. B; VEERKAMP, J. S. Influence of parental presence on the child's perception of, and behavior, during dental treatment. European Archives of Paediatric Dentistry, v.12, n. 4, p. 200-4, 2011.

FENLON, W. L.; DABBS, A. R.; CURZON, M. E. J. Parental presence during treatment of the child patient: a study with British parents. British Dental Journal, v. 174, n. 1, p. 174-23, 1993.

FRANKL, S. N.; SHIERE, F. R.; FOGELS, H. R. Should the parent remain with the child in the dental operatory? ASDC Journal of Dental for Children, v. 29, n. 1, p.150-163, 1962.

KLINGBERG, G.; BROBERG, A. G. Dental fear/anxiety and dental behavior management problems in children and adolescents: a review of prevalence and 
concomitant psychological factors. International Journal of Paediatric Dentistry, v. 17, n. 6, p. 391-406, 2007.

KOTSANOS, N.; ARHAKIS, A.; COOLIDGE, T. Parental presence versus absence in the dental operatory: a technique to manage the uncooperative child dental patient.

European Journal of Paediatric Dentistry, v. 6, n. 3, p.144-48, 2005,

KOTSANOS, N.; COOLIDGE, T.; VELONIS, D.; ARAPOSTATHIS, K. N. A form of 'parental presence/absence' (PPA) technique for the child patient with dental behaviour management problems. European Archives of Paediatric Dentistry, v. 10, n. 2, p. 9092, 2009.

SATO, C. M.; CORRÊA, M. S. N. P. Odontopediatria na primeira infância. In: Gestão comportamental da criança e do seu núcleo familiar: A chave para o sucesso no atendimento odontopediátrico. São Paulo: Quintessence; 2017. p.199-220.

SHARAT, C.; GHAZI, S.; ABDULRAHMAN, A.; MOHAMMAD, A. Objective assessment of the influence of the parental presence on the fear and behavior of anxious children during their first restorative dental visit. Journal of International Social Preventive and Community Dentistry, v. 6, supl. 2. p.148-152, 2016.

VASILIKI, B.; KONSTANTINOS, A.; VASSILIS, K.; NIKOLAOS, K.; VAN LOVEREN, C. The effect of parental presence on the child's perception and cooperation during dental treatment. European Archives of Paediatric Dentistry, v. 17, n. 5, p. 381-386, 2016.

VENHAM, L. L.; GAULIN-KREMER, E.; MUNSTER, E.; BENGSTON-AUDIA, D.; COHAN, I. Interval rating scales for children's dental anxiety and uncooperative behavior. Pediatric Dentistry, v. 2, n.3, p.195-202, 1980.

WONG, D. L.; BAKER, C. M. Pain in children: comparison of assessment scales. Pediatric Nursery, v. 14, n. 1, p. 9-17, 1988. 